\title{
Formation of the Nicotinic Acetylcholine Receptor Binding Sites
}

\author{
William N. Green and Christian P. Wanamaker \\ Department of Pharmacological and Physiological Sciences, University of Chicago, Chicago, Illinois 60637
}

Nicotinic acetylcholine receptors (AChRs) are activated by ACh binding to two sites located on different $\alpha$ subunits. The two $\alpha$ subunits, $\alpha_{\gamma}$ and $\alpha_{\delta}$, are distinguished by their interface with $\gamma$ and $\delta$ subunits. We have characterized the formation of the ACh binding sites and found, contrary to the current model, that the sites form at different times and in a set order. The first site forms on $\alpha_{\gamma}$ subunits during the process of subunit assembly. Our data are consistent with the appearance of this site on $\alpha \beta \gamma \delta$ subunit tetramers soon after the site for the competitive antagonist $\alpha$-bungarotoxin has formed and $\delta$ subunits have assembled with $\alpha \beta \gamma$ trimers. The second site is located on $\alpha_{\delta}$ subunits and forms after AChR subunits have assembled into $\alpha_{2} \beta \gamma \delta$ pentamers. By determining the order in which the ACh binding sites form, we have also identified the sites in which the $\delta$ and second $\alpha$ subunits associate during subunit assembly.

Key words: protein folding and assembly; acetylcholine; $\alpha$-bungarotoxin; nicotinic receptors; pharmacology; ligand binding sites
The binding of different ligands to a diverse set of membrane receptors is the primary mechanism by which information is transferred across cell membranes. For many different membrane receptors, much progress has been made in determining the structural details of ligand binding to its receptor. However, almost nothing is known about the protein folding and assembly events that give rise to these binding sites. Using the muscle-type nicotinic acetylcholine receptor (AChR), we have examined how the ACh binding sites form on this class of receptors. These receptors are composed of four distinct yet homologous subunits, $\alpha, \beta, \gamma$, and $\delta$, which assemble into AChR $\alpha_{2} \beta \gamma \delta$ pentamers. The muscle-type AChR is the best characterized member of a family of neurotransmitter-gated ion channels that includes neuronal AChRs, 5- $\mathrm{HT}_{3}$ receptors, glycine receptors, and $\mathrm{GABA}_{\mathrm{A}}$ receptors. As such, the muscle-type AChR has long served as a model system for the other receptors in the family (for review, see Unwin, 1993; Changeux, 1995; Karlin and Akabas, 1995; Lindstrom, 1995).

Several small ligands bind to AChRs. These ligands include agonists such as nicotine and the neurotransmitter ACh and small competitive antagonists such as curare. Amino acid residues located within three different regions of the N-terminal, extracellular domain of the $\alpha$ subunits have been identified as residues at the ACh binding sites. Other experiments have identified additional amino acids on the $\gamma$ and $\delta$ subunits that contribute to the binding sites (for review, see Galzi and Changeux, 1994; Karlin and Akabas, 1995; Tsigelny et al., 1997). Furthermore, for the ACh binding site to form, disulfide bonding and N-linked glycosylation within this $\mathrm{N}$-terminal $\alpha$ subunit domain appear to be

\footnotetext{
Received Feb. 24, 1998; revised May 5, 1998; accepted May 8, 1998.

This work was funded by grants from the National Institute of Health, National Science Foundation and the Brain Research Institute to W.N.G. We are most grateful to Dr. R. Fairclough for mAb 247g, Dr. J. Lindstrom for mAb 14, the McNamee lab for help in preparing the bromo-ACh affinity resin, and Dr. T. Claudio for the cell line stably expressing the four Torpedo AChR subunits and the anti-Bgt antiserum. We also thank Dr. Michael Gertz for help during the early stages of this work and Dr. R. Fairclough and other members of the Green laboratory for discussion and comments about this paper.

Correspondence should be addressed to Dr. William N. Green, Department of Pharmacological and Physiological Sciences, University of Chicago, 947 East 58th Street, Chicago, IL 60637.

Copyright (ㄷ) 1998 Society for Neuroscience $\quad 0270-6474 / 98 / 185555-10 \$ 05.00 / 0$
}

necessary (Mishina et al., 1985). The formation of the ACh sites, thus, requires the coordination of several post-translational events that draw together different regions of the $\alpha$ subunit $\mathrm{N}$-terminal domain, and assemble the $\alpha$ subunits with the other subunits.

Processing, folding, and assembly of AChR subunits are slow events, taking $\sim 2 \mathrm{hr}$ to complete (Merlie and Lindstrom, 1983). Despite the slow kinetics of these events, intermediates with $\mathrm{ACh}$ binding sites have been difficult to isolate. ACh binding sites form when $\alpha$ subunits are expressed heterologously with either the $\gamma$ or $\delta$ subunits in the absence of the other two subunits (Blount and Merlie, 1989). Based on these findings, it was proposed that the two ACh binding sites form on either $\alpha \gamma$ or $\alpha \delta$ heterodimers at the same time during the early stages of subunit assembly (Blount et al., 1990; Gu et al., 1991; Saedi et al., 1991). In this paper, we have characterized the formation of the ACh binding sites using cells expressing all four AChR subunits and present data at odds with the current model describing ACh binding site formation. We find that the first ACh binding site forms on $\alpha_{\gamma}$ subunits during subunit assembly and that the second site, located on the $\alpha_{\delta}$ subunit, forms after AChR subunits have assembled into $\alpha_{2} \beta \gamma \delta$ pentamers. The data provide evidence for the subunit arrangement of assembly intermediates and for the location of the subunit interfaces in which the unassembled $\delta$ and second $\alpha$ subunits assemble.

\section{MATERIALS AND METHODS}

Metabolic labeling and subunit precipitations. Mouse L fibroblasts, stably transfected with the Torpedo subunit cDNAs under the control of SV40 promoters (Claudio et al., 1987), were maintained in DMEM plus 10\% calf serum and HAT $(15 \mu \mathrm{g} / \mathrm{ml}$ hypoxanthine, $1 \mu \mathrm{g} / \mathrm{ml}$ aminopterin, and $5 \mu \mathrm{g} / \mathrm{ml}$ thymidine) in $5 \% \mathrm{CO}_{2}$. To enhance subunit expression, the DMEM was supplemented with $20 \mathrm{~mm}$ sodium butyrate (NB medium) $36 \mathrm{hr}$ before the experiment. Cultures in $10 \mathrm{~cm}$ plates were labeled as described previously (Green and Claudio, 1993; Green and Wanamaker, 1997). Briefly, cultures were pulse-labeled in $2 \mathrm{ml}$ of methionine- and cysteine-free NB medium, supplemented with $333 \mu \mathrm{Ci}$ of a ${ }^{35} \mathrm{~S}$ methionine ${ }^{35} \mathrm{~S}$-cysteine mixture (NEN EXPE ${ }^{35} \mathrm{~S}^{35} \mathrm{~S}$ ). The labeling was stopped with the addition of DMEM plus $5 \mathrm{mM}$ methionine and cysteine. To follow the subsequent changes in the labeled subunits, the cells were "chased" by incubation for the indicated times in NB medium at $20^{\circ} \mathrm{C}$. The cells were solubilized in (in mM): $150 \mathrm{NaCl}, 5$ EDTA, 50 Tris, $\mathrm{pH}$ 
7.4, $2 \mathrm{PMSF}$, and $2 \mathrm{~N}$-ethylmaleimide, plus $0.02 \% \mathrm{NaN}_{3}, 1.83 \mathrm{mg} / \mathrm{ml}$ phosphatidylcholine, and $1 \%$ Lubrol. Solubilized AChR subunits were purified on an acetylcholine affinity column, precipitated with a slurry of bungarotoxin (Bgt)-Sepharose and/or immunoprecipitated with anti-Bgt polyclonal antiserum or the conformation-dependent monoclonal antibodies (mAbs) 14 (Tzartos and Lindstrom, 1980), 35 (American Type Culture Collection, Manassas, VA) or 247g (Mihovilovic and Richman, 1987). For the labeled AChR complexes immunoprecipitated with antiBgt polyclonal antiserum (see Figs. $3 A, 4 B$ ), the complexes were first bound with Bgt. In Figure $3 A, 10 \mathrm{~nm}$ cold Bgt was added to each of the gradient fractions and maintained at $4^{\circ} \mathrm{C}$ rotated overnight before the immunoprecipitation. In Figure $4 A, B \quad 10 \mathrm{nM}{ }^{125} \mathrm{I}$-Bgt was added to the chase medium for the length of the chase time, after which the cells were solubilized. Bromo-ACh affinity resin was prepared by a scaleddown version of a previously described protocol (Chak and Karlin, 1992), and Bgt-Sepharose was prepared by coupling Bgt to cyanogen bromideactivated Sepharose according to the manufacturer's directions (Pharmacia, Piscataway, NJ).

The bromo-ACh affinity column does not quantitatively bind all $\mathrm{ACh}$ binding sites. Compared with Bgt-Sepharose precipitation or immunoprecipitating Bgt-bound complexes with anti-Bgt antibodies, the bromoACh affinity protocol isolated $30-40 \%$ of the total subunit complexes (compared with Bgt-Sepharose), which is not surprising given the relatively weak affinity of ACh to its binding site. In contrast, Bgt-Sepharose precipitation, immunoprecipitation of Bgt-bound complexes with antiBgt antibodies, and immunoprecipitation with mAb 247g all appear to precipitate most available complexes.

Antibody-subunit complexes were precipitated with protein G-Sepharose and electrophoresed on 7.5\% SDS polyacrylamide gels, fixed, treated 30 min with Amplify (Amersham, Arlington Heights, IL) to enhance the signal, dried on a gel dryer, and exposed to film at $-70^{\circ} \mathrm{C}$ with an intensifying screen. Autoradiographs were quantified by scanning densitometry using a flatbed scanner and analyzed with the Intelligent Quantifier software from Bioimage. A standard, provided by Bioimage, with 21 bands ranging from 0.05 to 3.05 optical density units in 0.15 increments was used to calibrate the linearity of the densitometer before using it. To ensure that quantified bands were in the linear range and the darker signals were not saturated, three to five exposures were taken of each autoradiograph, and each was scanned to ensure that the scanned bands remained in the linear range of the film.

${ }^{125} I-\alpha$-Bgt binding and inhibition by curare. To measure cell surface ${ }^{125} \mathrm{I}$-Bgt binding, cells grown at $20^{\circ} \mathrm{C}$ for $48 \mathrm{hr}$ were incubated at room temperature in PBS containing $4 \mathrm{nM}{ }^{125} \mathrm{I}-\mathrm{Bgt}(140-170 \mathrm{cpm} / \mathrm{fmol})$ for 2 $\mathrm{hr}$ with or without mAb $247 \mathrm{~g}$. Cultures were washed and scraped off the plates, and the cell surface counts were determined by gamma counting.

To measure the inhibition of intracellular ${ }^{125} \mathrm{I}-\mathrm{Bgt}$ binding by $d$-tubocurarine (dTC), ${ }^{125}$ I-Bgt binding was measured after solubilization of the cells as described previously (Wang and Claudio, 1993). Cell surface Bgt binding sites were blocked with cold Bgt, after which the cells were solubilized and AChR subunit complexes were immunoprecipitated with the indicated antibody. ${ }^{125} \mathrm{I}$-Bgt binding was assayed with the AChR subunit complexes bound to antibody and protein G-Sepharose. The protein G-Sepharose pellets were incubated in the indicated dTC concentration for $15 \mathrm{~min}$ before the addition of ${ }^{125} \mathrm{I}$-Bgt. Ten nanomolar ${ }^{125} \mathrm{I}$-Bgt was added to the pellets and incubated at room temperature for $15 \mathrm{~min}$ to measure the initial rate of Bgt binding. The pellets were washed, and the counts were determined by gamma counting. The line through the data represents a least squares fit to the equation: fraction of maximum ${ }^{125} \mathrm{I}$-Bgt bound $=A\left(1 /\left(1+\left([\mathrm{dTC}] / \mathrm{IC}_{50}{ }^{1}\right)\right)+B(1 /(1+([\mathrm{dTC}] /\right.$ $\left.\mathrm{IC}_{50}{ }^{2}\right)$ ), where $\mathrm{IC}_{50}{ }^{1}$ and $\mathrm{IC}_{50}{ }^{2}$ are $\mathrm{IC}_{50}$ values for two separate binding sites, and $A$ and $B$ are the fractions of total sites for each of the two sites.

Sucrose gradients. Cell lysates were layered on a $5 \mathrm{ml} \mathrm{5-20 \%} \mathrm{linear}$ sucrose gradient prepared in the lysis buffer. Gradients were centrifuged in a Beckman SW 50.1 rotor at 40,000 rpm for $14.25 \mathrm{hr}\left(\omega^{2} \mathrm{t}=9.0 \times\right.$ $\left.10^{11}\right)$. Three hundred-microliter fractions were collected from the top of the gradient. The linearity of the gradient was confirmed by measuring the osmolality of each fraction of some of the gradients.

\section{RESULTS}

\section{Formation of ACh binding sites}

In this study, we have characterized the formation of the ACh binding sites using cells stably expressing all four Torpedo AChR subunits (Claudio et al., 1987). Torpedo AChR subunit folding and assembly is temperature-sensitive, which allows us to isolate and characterize assembly intermediates because the kinetics of AChR subunit assembly are slowed by more than an order of magnitude at $20^{\circ} \mathrm{C}$ (Green and Claudio, 1993; Green and Wanamaker, 1997). AChR subunits, pulse-labeled with ${ }^{35} \mathrm{~S}$-methionine and -cysteine, were first purified on a bromo-ACh affinity column and then immunoprecipitated with $\alpha$ subunit-specific mAb 35 (Fig. $1 A, B$ ) or with $\gamma$ and $\delta$ subunit-specific mAb 88b (Fig. $1 B$ ). No labeled subunits were isolated immediately after the pulse label using this bromo-ACh affinity protocol in Figure $1 A, B$, even though both unassembled and assembled $\alpha$ subunits were present in the cell lysate. The presence of labeled AChR subunits immediately after the pulse label was shown by the mAb 35 precipitation of labeled subunits (Fig. $1 C$ ). The ACh binding sites isolated by the bromo-ACh affinity protocol appear a significant amount of time after the synthesis of the subunits (Fig. $1 A, B$, at 3, 6, and $12 \mathrm{hr}$ ). The time course of the formation of the ACh binding sites is shown quantitatively from the scanning densitometry of this and other experiments (Fig. $1 B$ ). Initially, the subunits isolated by the bromo-ACh affinity protocol had a $\sim 1: 1: 1: 1$ ratio of $\alpha: \beta: \gamma: \delta$ subunits (Fig. $1 B$, at 3, 6, and 12 hr). If ACh binding sites existed on unassembled $\alpha$ subunits, we would have expected a larger ratio of $\alpha$ to the other subunits. Also, we would expect differences in the results, which were not observed, when the $\gamma$ and $\delta$ subunitspecific mAb was used instead of the $\alpha$ subunit-specific mAb 35 after the bromo-ACh affinity purification (Fig. $1 B$ ). In agreement with previous studies (Blount and Merlie, 1988; Paulson et al., 1991), we conclude, therefore, that no ACh binding sites appear on unassembled $\alpha$ subunits, and ACh binding sites form on complexes containing $\alpha$ and other AChR subunits.

An important feature of the time course of ACh binding site formation was that, $12 \mathrm{hr}$ after subunit labeling, the number of $\alpha$ subunits in the isolated complexes continued to increase and eventually more than doubled whereas the number of $\beta, \gamma$, and $\delta$ subunits remained constant (Fig. $1 A, B$ ). We have found previously that the ratio of $\alpha$ subunit doubles relative to the other subunits as a second $\alpha$ subunit assembles with the other subunits to form $\alpha_{2} \beta \gamma \delta$ pentamers (Green and Claudio, 1993). The doubling of $\alpha$ subunits relative to the other subunits, thus, indicates that the AChR subunit complexes isolated by the bromo-ACh affinity protocol initially contain a single $\alpha$ subunit. Based on the time course shown in Figure $1 B$, subunit complexes with $\mathrm{ACh}$ binding sites isolated $3-12 \mathrm{hr}$ after subunit labeling have a single $\alpha$ subunit, and the second $\alpha$ subunit is added $12-48 \mathrm{hr}$ after subunit labeling. Because $\alpha$ subunits can have at most a single $\mathrm{ACh}$ binding site, one of the two ACh binding sites is present before the addition of the second $\alpha$ subunit. Furthermore, because ACh binding sites do not form on unassembled $\alpha$ subunits, the second $\mathrm{ACh}$ binding site must appear after the addition of the second $\alpha$.

\section{Formation of Bgt binding sites}

Previous studies suggested that the formation of the binding sites for Bgt, a high-affinity competitive antagonist of ACh, precedes the formation of the ACh binding sites (Merlie and Lindstrom, 1983; Blount and Merlie, 1988; Paulson et al., 1991). To test when the Bgt binding sites form relative to the ACh binding sites, labeled AChR subunits were isolated using Bgt-Sepharose (Fig. $2 A$ ), and the results of this and other experiments were quantified by densitometry (Fig. 2B). Many features of Bgt binding site formation are similar to ACh binding site formation. As with the bromo-ACh affinity purification, no labeled AChR subunits were precipitated by Bgt-Sepharose immediately after the subunit la- 
A.

\section{BromoACh Affinity Resin}

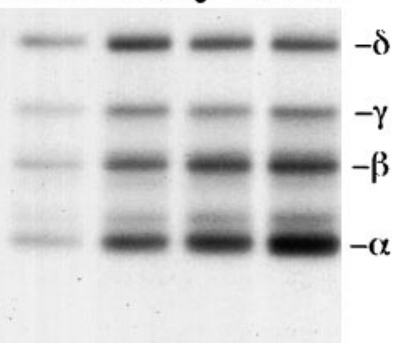

Chase times: $\begin{array}{lllllll}0 & 3 & 6 & 12 & 24 & 48 & \text { hrs. }\end{array}$

C.

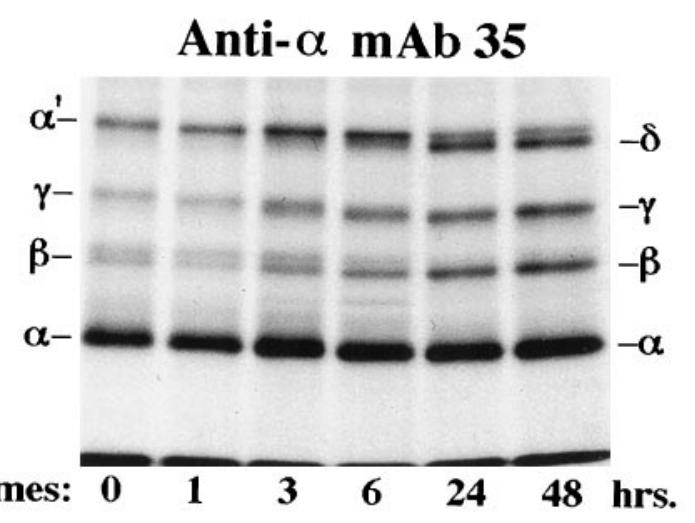

\section{Chase times: $\begin{array}{lllllllll} & 0 & 1 & 3 & 6 & 24 & 48 & \text { hrs. }\end{array}$}

B.

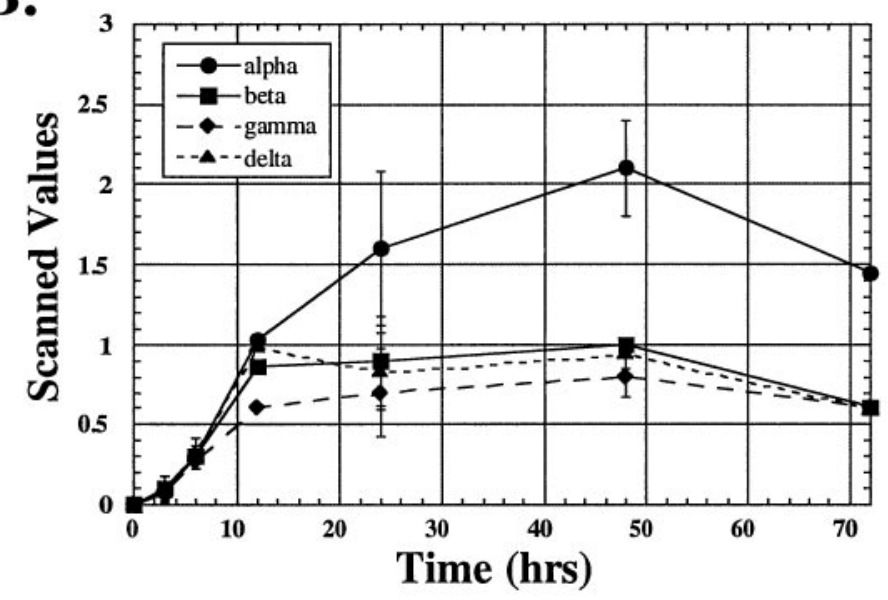

Figure 1. Formation of the ACh binding sites. A, Purification of labeled AChR subunits with bromo-ACh affinity gel. Displayed is an autoradiogram of the labeled subunits analyzed on a 7.5\% SDS-PAGE gel. Mouse L fibroblasts, stably expressing all four Torpedo AChR subunits (Claudio et al., 1987), were pulse-labeled with a ${ }^{35} \mathrm{~S}$-methionine-cysteine mixture for $30 \mathrm{~min}$ at $37^{\circ} \mathrm{C}$ and chased for the indicated times at $20^{\circ} \mathrm{C}$. Cell lysates were passed through a bromo-ACh affinity column two times; the subunits were eluted from the column and immunoprecipitated with the $\alpha$ subunit-specific mAb 35. Similar results were obtained when the bromo-ACh affinity-purified subunits were immunoprecipitated with the $\gamma$ and $\delta$ subunit-specific mAb 88b instead of mAb 35 (Fig. $1 B$ ). B, Scanning densitometry of the bromo-ACh affinity-purified AChR subunits. The time course of the formation of the ACh binding site is shown quantitatively from the scanned values of the experiment shown in $A$ and other experiments in which mAb 35 or mAb $88 \mathrm{~b}$ were used to precipitate the subunits eluted from the bromo-ACh affinity column. No differences in the results were observed when mAb $88 \mathrm{~b}$ was substituted for $\mathrm{mAb} 35$. All scanned values are displayed as the fraction of the $\beta$ subunit value at $48 \mathrm{hr}$. The $0,6,24$, and 48 hr time points represent the mean \pm SD for five separate experiments. The $3 \mathrm{hr}$ time point represents the mean \pm SD for three separate experiments, and the 12 and $72 \mathrm{hr}$ time points represent the mean for two separate experiments. At the $6 \mathrm{hr}$ time point, the values for the subunits were $\alpha, 0.30 \pm 0.07 ; \beta, 0.30 \pm 0.04 ; \gamma, 0.27 \pm 0.05$; and $\delta, 0.32 \pm 0.10$, yielding a $\alpha: \beta: \gamma: \delta$ subunit ratio of 1.0:1:0.9:1.1. Taking into account differences in subunit cysteine and methionine content and the ratio of ${ }^{35} \mathrm{~S}$-methionine and ${ }^{35} \mathrm{~S}$-cysteine during labeling of the subunits, the subunit ratio should be 1.3:1.0:0.8:1.0 for a subunit stoichiometry of 1:1:1:1. At the $48 \mathrm{hr}$ time point, the values were $\alpha, 2.1 \pm 0.3 ; \beta, 1.0 ; \gamma, 0.80 \pm 0.13$; and $\delta, 0.94 \pm 0.09$, and the ratio of the $\alpha$ subunit to the other subunits doubled from the 6 to the $48 \mathrm{hr}$ time point. $C$, Immunoprecipitation of labeled subunits with $\alpha$ subunit-specific mAb 35. Cells were treated as in $A$ except that immunoprecipitation using mAb 35, which recognizes both unassembled and assembled $\alpha$ subunits (Green and Claudio, 1993), replaced the bromo-ACh affinity purification step. The band labeled $\alpha^{\prime}$ has previously been shown to be different from the $\delta$ subunit and related to the $\alpha$ subunit, although it migrates just above the $\delta$ subunit (Green and Claudio, 1993; Green and Wanamaker, 1997).

bel. The AChR subunits initially precipitated by Bgt-Sepharose had a $\sim 1: 1: 1: 1$ ratio of $\alpha: \beta: \gamma: \delta$ subunits (Fig. $2 B$, at 3,6 , and 12 hr), and the ratio of $\alpha$ to the other subunits doubled by the $48 \mathrm{hr}$ time point.

Although the time course of Bgt binding site formation was similar to that of ACh binding site formation, there were differences at the 6 and $24 \mathrm{hr}$ time points. During the formation of both binding sites, the $12 \mathrm{hr}$ time point is the point at which the number of $\beta, \gamma$, and $\delta$ subunits saturated and after which the number of $\alpha$ subunits doubled. As discussed above, these data indicate that the formation of the first $\mathrm{ACh}$ and $\mathrm{Bgt}$ binding sites occurs during the first $12 \mathrm{hr}$ on complexes containing a single $\alpha$ subunit, whereas the second binding sites form in the period between the 12 and $48 \mathrm{hr}$ time points after the addition of a second $\alpha$ subunit. For the Bgt-Sepharose precipitation at the $6 \mathrm{hr}$ time point, the scanned values (mean \pm SD for six gels) were $0.71 \pm 0.28,0.57 \pm 0.31,0.50 \pm 0.18$, and $0.62 \pm 0.20$ for the $\alpha$, $\beta, \gamma$, and $\delta$ subunits, respectively. For bromo-ACh affinity purification at the $6 \mathrm{hr}$ time point, the normalized scanned values (mean \pm SD for five gels) were half that of the Bgt-Sepharose precipitation: $0.30 \pm 0.07,0.30 \pm 0.04,0.27 \pm 0.05$, and $0.32 \pm$ 0.10 for the $\alpha, \beta, \gamma$, and $\delta$ subunits, respectively. These data suggest that formation of the first ACh binding site lags behind formation of the first Bgt binding site. Furthermore, at the $24 \mathrm{hr}$ time point the scanned value of the Bgt-Sepharose $\alpha$ subunit was $2.1 \pm 0.7$ compared with $1.6 \pm 0.48$ for the bromo-ACh affinitypurified $\alpha$ subunit. Again, the ACh binding site, in this case the second site, appears to form after the second Bgt binding site.

\section{The first ACh site forms on $\alpha \beta \gamma \delta$ tetramers after the Bgt binding site forms}

In an earlier study, we presented data that Bgt binding sites and the conformation-dependent epitope to mAb 14 first appear on $\alpha \beta \gamma$ trimers just before the addition of $\delta$ subunits to the trimers 
A.

\section{Bgt-Sepharose}
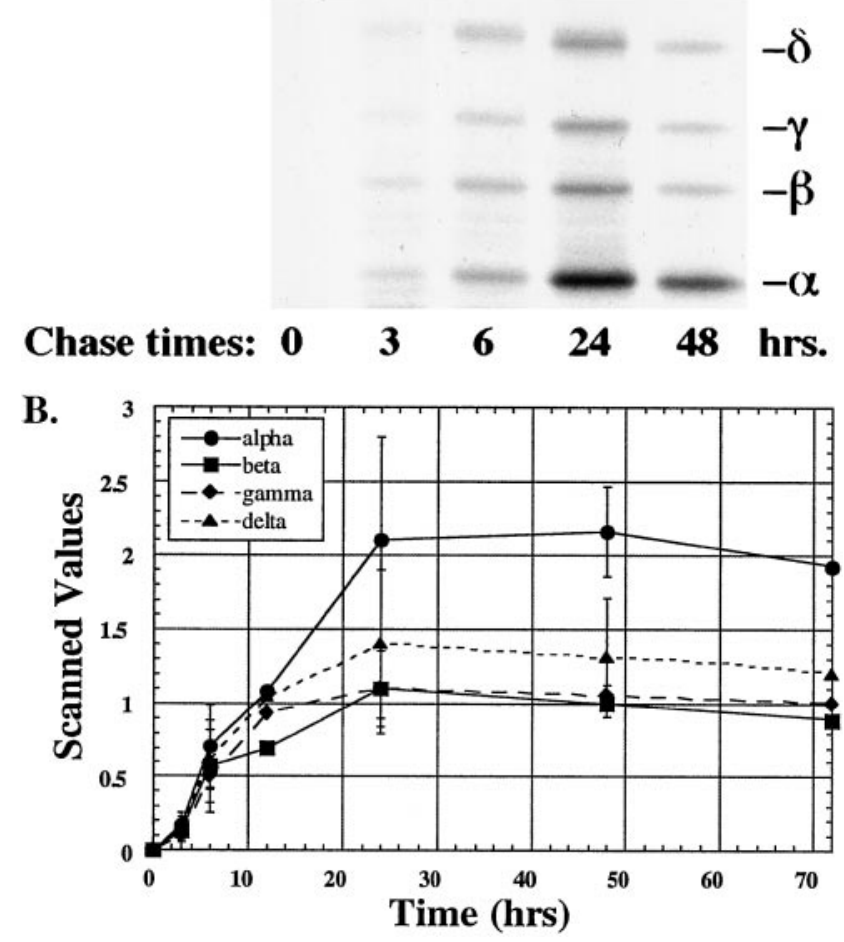

Figure 2. Formation of the Bgt binding sites. $A$, Purification of labeled AChR subunits with Bgt-Sepharose. Cells were treated as in Figure $1 A$, except that precipitation using Bgt-Sepharose replaced the bromo-ACh affinity purification steps. $B$, Scanning densitometry of AChR subunits precipitated by Bgt-Sepharose. The time course of the formation of Bgt binding sites is shown quantitatively from the scanned values of the experiment shown in $A$ and other experiments. All scanned values are displayed as the fraction of the $\beta$ subunit value at $48 \mathrm{hr}$. The $0,6,24$, and $48 \mathrm{hr}$ time points represent the mean $\pm \mathrm{SD}$ for six separate experiments. The $3 \mathrm{hr}$ time point represents the mean \pm SD for five separate experiments, and the 12 and $72 \mathrm{hr}$ time points represent the mean for two separate experiments. At the $6 \mathrm{hr}$ time point, the values for subunits were $\alpha, 0.71 \pm 0.28 ; \beta, 0.57 \pm 0.31 ; \gamma, 0.50 \pm 0.18 ;$ and $\delta, 0.62 \pm 0.20$, yielding an $\alpha: \beta: \gamma: \delta$ subunit stoichiometry of $1.2: 1: 0.87: 1.1$. At the $48 \mathrm{hr}$ time point, the values were $\alpha, 2.2 \pm 0.3 ; \beta, 1.0 ; \gamma, 1.0 \pm 0.07$; and $\delta, 1.3 \pm 0.5$. As with the bromo-ACh affinity purification, the ratio of the $\alpha$ subunit to the other subunits doubled from the 6 to the $48 \mathrm{hr}$ time point. The reason that the ratio of the $\delta$ subunit to the other subunits is somewhat larger than expected is attributable to the occasional presence of a contaminating band, $\alpha^{\prime}$, related to the $\alpha$ subunit that migrates on gels close to the $\delta$ subunit (Fig. 1C) and will merge with the $\delta$ subunit.

to assemble $\alpha \beta \gamma \delta$ tetramers (Green and Claudio, 1993). If ACh binding sites form after Bgt binding sites, it raises the possibility that the first ACh binding site forms on $\alpha \beta \gamma \delta$ tetramers. To characterize in more detail the subunit complexes on which the first ACh and Bgt binding sites form, cells were solubilized $6 \mathrm{hr}$ after the pulse label, and labeled subunits were size-fractionated on sucrose gradients before isolation (Fig. 3). After the sucrose gradient, subunit complexes were isolated by three different methods. In Figure $3 A$, complexes were bound by Bgt and isolated by anti-Bgt antibodies. In Figure $3 B$, complexes were isolated by bromo-ACh affinity purification. In Figure $3 C$, complexes were isolated by $\mathrm{mAb} 14$ immunoprecipitation. Bgt binding sites as well as the conformation-dependent mAb 14 epitope are evident on complexes that migrate at $6-7 \mathrm{~S}$ and that contain predominantly $\alpha, \beta$, and $\gamma$ subunits (Fig. $3 A, C$, boxed fractions), consistent with the presence of these sites on $\alpha \beta \gamma$ trimers. The $\alpha \beta \gamma$ trimers are

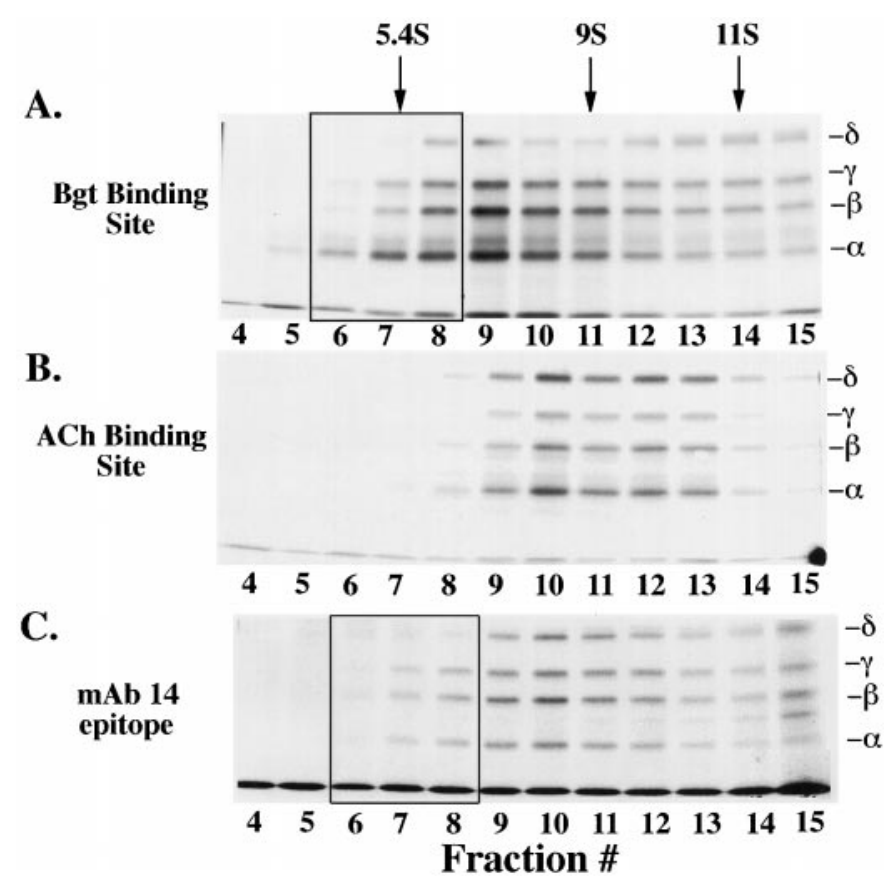

Figure 3. The first ACh site forms just after the Bgt binding site forms on $\alpha \beta \gamma \delta$ tetramers. $A-C$, Sedimentation of AChR subunit complexes containing Bgt binding sites $(A)$, ACh binding sites $(B)$, or the $\mathrm{mAb} 14$ epitope $(C)$. Cells were pulse-labeled with ${ }^{35} \mathrm{~S}$-methionine-cysteine for $30 \mathrm{~min}$ at $37^{\circ} \mathrm{C}$ and cultured for $6 \mathrm{hr}$ at $20^{\circ} \mathrm{C}$. Solubilized subunits were fractionated on a 5-20\% linear sucrose gradient. AChR subunits in gradient fractions $4-15$ were precipitated by three different methods. In $A$, Bgt-bound subunit complexes were precipitated using anti-Bgt antibodies. In $B$, subunit complexes were bromo-ACh affinity-purified as in Figure $1 A$. In $C$, subunit complexes were immunoprecipitated with conformation-dependent mAb 14. The arrows above $A$ mark the peak fractions of each of three standards: alkaline phosphatase (5.4S), cell surface Torpedo AChRs (9S, as in Fig. 4C), and catalase (11S), which were run on parallel gradients. Consistent with the results in Figures $1 A$ and $2 A$, the majority of subunit complexes precipitated $6 \mathrm{hr}$ after the ${ }^{35} \mathrm{~S}$ methionine-cysteine label are $\alpha \beta \gamma \delta$ tetramers. This conclusion is based on the $\sim 1: 1: 1: 1$ stoichiometry of $\alpha: \beta: \gamma: \delta$ subunits and the fact that the peak fractions of all three gradients occur at fractions 9 and 10 or at $\sim 8 \mathrm{~S}$, which is where $\alpha \beta \gamma \delta$ tetramers would be expected to sediment (Green and Claudio, 1993). A shoulder to this peak is observed at fractions 6-8 on the Bgt binding site and mAb 14 epitope gradients (highlighted by the boxes) but not on the ACh binding site gradient. Complexes containing predominantly $\alpha, \beta$, and $\gamma$ subunits are observed in this shoulder, which migrate at $6-7 \mathrm{~S}$ and appear to be $\alpha \beta \gamma$ trimers. Although many of the trimers and tetramers migrate where expected, large numbers of both complexes extend farther into the gradient than expected. We do not fully understand the broad size distribution displayed by trimer and tetramers. However, this property has been observed previously for the intracellular AChR complexes, both for the Torpedo subunits at reduced temperature (Ross et al., 1991; Saedi et al., 1991; Green and Wanamaker, 1997) and for the mouse subunits at $37^{\circ}$ (Blount et al., 1990; Gu et al., 1991; Green and Claudio, 1993; Kreienkamp et al., 1995).

absent when the labeled subunits are isolated using bromo-ACh affinity purification (Fig. 3B).

The properties of the bromo-ACh affinity-purified subunit complexes in Figure $3 B$ are consistent with $\alpha \beta \gamma \delta$ tetramers. These complexes contain all four subunits with a $\sim$ 1:1:1:1 stoichiometry among the subunits, and the peak fractions for these complexes, fractions 9 and 10 , migrate at $\sim 8 \mathrm{~S}$, between where trimers at $6-7 \mathrm{~S}$ and surface $\alpha_{2} \beta \gamma \delta$ pentamers at $9 \mathrm{~S}$ migrate on the gradient. The properties of the Bgt-Sepharose-precipitated subunit complexes in Figure $3 A$ are consistent with a combination of $\alpha \beta \gamma$ trimers and $\alpha \beta \gamma \delta$ tetramers. In the $6-7 \mathrm{~S}$ region, there are 
predominantly $\alpha \beta \gamma$ trimers. At the $8-9 \mathrm{~S}$ region, the complexes have a subunit stoichiometry of $\sim 1: 1: 1: 0.6$ for $\alpha: \beta: \gamma: \delta$, attributed to the presence of both trimers and tetramers. In contrast, in the $10-11 \mathrm{~S}$ region, the subunit stoichiometry is $\sim 1: 1: 1: 1$, consistent with only tetramers. The results with the gradients indicate that the first ACh binding site, unlike the Bgt binding site, does not form on $\alpha \beta \gamma$ trimers. Instead, the first ACh binding site forms on $\alpha \beta \gamma \delta$ tetramers after the formation of the first Bgt binding site and addition of the $\delta$ subunit to $\alpha \beta \gamma$ trimers.

\section{The second ligand binding sites form on $\alpha_{2} \beta \gamma \delta$ pentamers that are transported to the surface}

Up to $12 \mathrm{hr}$ after the pulse label, both the Bgt and ACh binding sites exist on subunit complexes with a $\sim 1: 1: 1: 1$ stoichiometry of $\alpha: \beta: \gamma: \delta$ subunits. In the later stages of ligand binding site formation, the AChR complexes purified by Bgt-Sepharose and bromoACh affinity resin undergo the same change in which the amount of $\alpha$ subunit doubles (Figs. $1 B, 2 B$ ). If the doubling of the $\alpha$ subunit is actually a measure of the addition of a second $\alpha$ subunit during assembly of $\alpha_{2} \beta \gamma \delta$ pentamers, then the second ACh and Bgt binding sites must form after the assembly of $\alpha_{2} \beta \gamma \delta$ pentamers. To confirm that the doubling of the $\alpha$ subunit in Figure $2 B$ corresponds to the assembly of $\alpha_{2} \beta \gamma \delta$ pentamers, subunit complexes isolated using Bgt-Sepharose were size-fractionated on sucrose gradients. In Figure $4 A$, cells were solubilized $48 \mathrm{hr}$ after the pulse label, and cell surface AChRs were selectively removed from the cell lysate to obtain intracellular complexes (see Materials and Methods for details). The distribution of subunit complexes on the gradients has clearly changed during the period between $6 \mathrm{hr}$ (Fig. 3A) and $48 \mathrm{hr}$ (Fig. $4 A$ ). By $48 \mathrm{hr}$, the $\alpha \beta \gamma$ trimers observed at $6 \mathrm{hr}$ in the $6-7 \mathrm{~S}$ region have disappeared, and the majority of the complexes migrate in the $9 \mathrm{~S}$ region of the gradient, as shown by the scanned values displayed in Figure $4 D$. Cell surface receptors in these cells consist of a single population of $\alpha_{2} \beta \gamma \delta$ pentamers (Hartman et al., 1990), and the surface ${ }^{125}$ I-Bgt-bound AChRs, which were size-fractionated on parallel gradient, migrate precisely at 9S (Fig. 4C). In addition, the intracellular complexes at $48 \mathrm{hr}$ in the $9 \mathrm{~S}$ region have a subunit ratio of $\sim 2: 1: 1: 1$ for $\alpha: \beta: \gamma: \delta$ (see Fig. $4 D$ legend for details), which differs from that at $6 \mathrm{hr}$ in which the subunit ratios were consistent with $\alpha \beta \gamma$ trimers and $\alpha \beta \gamma \delta$ tetramers. These findings are all consistent with predominantly $\alpha_{2} \beta \gamma \delta$ pentamers precipitated by Bgt-Sepharose at $48 \mathrm{hr}$.

In Figure $4 B$, metabolically labeled, cell surface AChRs were also characterized on sucrose gradients. Our ability to isolate the labeled surface AChRs in Figure $4 B$ demonstrates that the labeled complexes purified by Bgt affinity methods (Fig. $2 A, B$ ) are transported to the cell surface. These AChR subunit complexes differ from the intracellular complexes (Fig. 4A,D) in several respects. The surface complexes (Fig. $4 B, E$ ) migrate in a tighter peak centered at $9 \mathrm{~S}$ on the sucrose gradient than the intracellular complexes. Almost all of the surface complexes are found in fractions 10, 11, and 12 of the gradient, whereas the intracellular complexes migrate in a broader peak encompassing fractions $9-14$. For the surface receptors, the subunit ratio is on average 2.7:1:0.7:0.9 (see Fig. $4 E$ legend for details). This subunit ratio is almost precisely the ratio obtained taking into account differences in subunit cysteine and methionine content and the ratio of ${ }^{35} \mathrm{~S}$-methionine and ${ }^{35} \mathrm{~S}$-cysteine during labeling, which is 2.7:1.0: $0.8: 1.0$ for a subunit stoichiometry of $2: 1: 1: 1$ for $\alpha: \beta: \gamma: \delta$. The ratio of the $\alpha$ to the other subunits is smaller for the intracellular complexes, ranging from $\sim 2$ for the complexes in the $9 \mathrm{~S}$ region (fractions 10-12) to $\sim 1$ in the $11 \mathrm{~S}$ region (fractions 13-15). The results indicate that the intracellular complexes include $\alpha \beta \gamma \delta$ tetramers with a 1:1:1:1 subunit ratio and a broad distribution across the gradient (fractions 9-15) as well as $\alpha_{2} \beta \gamma \delta$ pentamers at 9S. The presence of both pentamers and tetramers explains why the ratio of the $\alpha$ to the other subunits for intracellular complexes is $\sim 2$ at $48 \mathrm{hr}$ (Figs. $1 B, 2 B, 4 D$ ) as opposed to the expected value of 2.7. Altogether, our results characterizing subunit complexes on sucrose gradients (Figs. 3, 4) support our previous findings that AChR subunits assemble first as $\alpha \beta \gamma$ trimers, next into $\alpha \beta \gamma \delta$ tetramers, and finally into $\alpha_{2} \beta \gamma \delta$ pentamers, after which the complexes are transported to the cell surface (Green and Claudio, 1993; Green and Wanamaker, 1997).

\section{The first ACh binding site forms on the $\alpha_{\gamma}$ subunit}

Differences in the affinity of the two ACh binding sites for small ligands are caused by the association of the two $\alpha$ subunits with different subunits, either the $\gamma$ or $\delta$ subunits (Blount and Merlie, 1989; Pedersen and Cohen, 1990; Sine and Claudio, 1991). To test which of the two ACh binding sites forms first, the AChR antagonist dTC was used to inhibit the initial rate of ${ }^{125} \mathrm{I}-\mathrm{Bgt}$ binding (Fig. 5). dTC binds to the two ACh binding sites with $\sim 100$-fold difference in affinity (Neubig and Cohen, 1979; Sine and Taylor, 1981), and the high- and low-affinity sites are located on the $\alpha_{\gamma}$ and $\alpha_{\delta}$ subunits, respectively (Blount and Merlie, 1989; Pedersen and Cohen, 1990). When intracellular AChR complexes are isolated $48 \mathrm{hr}$ after the cells are shifted from 37 to $20^{\circ} \mathrm{C}$, the isolated complexes are predominately $\alpha_{2} \beta \gamma \delta$ pentamers (Fig. $4 B$ ). At this time, the inhibition by dTC is consistent with two different binding sites on the two $\alpha$ subunits with a 70-fold difference in affinity for dTC, as calculated by the fit to the data of Figure 5 (see also Claudio et al., 1987). Different results were obtained for AChR complexes isolated $6 \mathrm{hr}$ after the temperature shift, at which time assembled complexes consist of trimers and tetramers (Fig. 3; see Green and Claudio, 1993). The inhibition by dTC then was consistent with AChR complexes that predominantly contain a single binding site with high affinity for dTC (Fig. 5). The data together indicate that the first ACh binding site formed, the site found on $\alpha \beta \gamma \delta$ tetramers, is the high-affinity curare site located on the $\alpha_{\gamma}$ subunit.

As another test of which of the two ACh binding sites forms first, we obtained a conformational-dependent mAb, mAb $247 \mathrm{~g}$, which has been reported to specifically recognize and block the high-affinity dTC site on the $\alpha \gamma$ subunit (Mihovilovic and Richman, 1987). At saturating concentrations, mAb $247 \mathrm{~g}$ blocked $\sim 50 \%$ of the surface Bgt binding sites (Fig. 6A). Furthermore, when the inhibition of ${ }^{125} \mathrm{I}$-Bgt binding by dTC was measured for subunit complexes precipitated by mAb $247 \mathrm{~g} 48 \mathrm{hr}$ after the temperature shift, the inhibition by dTC was consistent with AChR complexes that predominantly contain a single binding site with the low affinity for dTC (Fig. 6B). Together, the data indicate that $\mathrm{mAb} 247 \mathrm{~g}$ binds to and blocks the binding site on the $\alpha_{\gamma}$ subunit but not the other binding site. When mAb $247 \mathrm{~g}$ was used to immunoprecipitate labeled subunits undergoing assembly (Fig. $6 C$ ), we obtained results similar to those obtained using the bromo-ACh (Fig. 1 $A$ ) and Bgt (Fig. 2A) affinity resin. This similarity is shown quantitatively in Figure $6 D$, in which the scanned values for all four subunits are displayed. These data demonstrate that the mAb $247 \mathrm{~g}$ epitope forms at approximately the same time as the first ACh site, well before the second ACh site forms, and is further evidence that the $\alpha_{\gamma} \mathrm{ACh}$ binding site is the first ACh binding site formed. 
A.

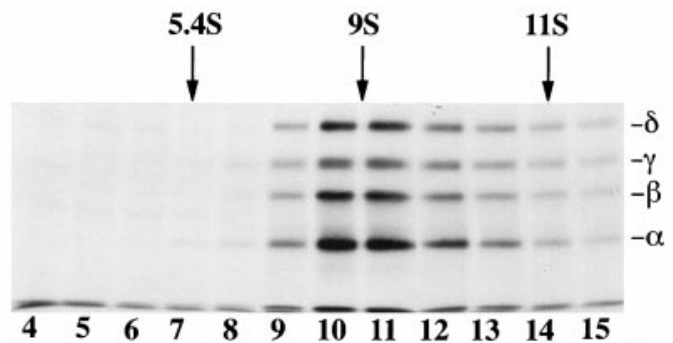

B.

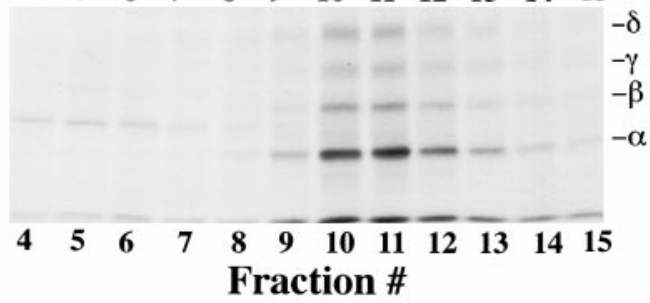

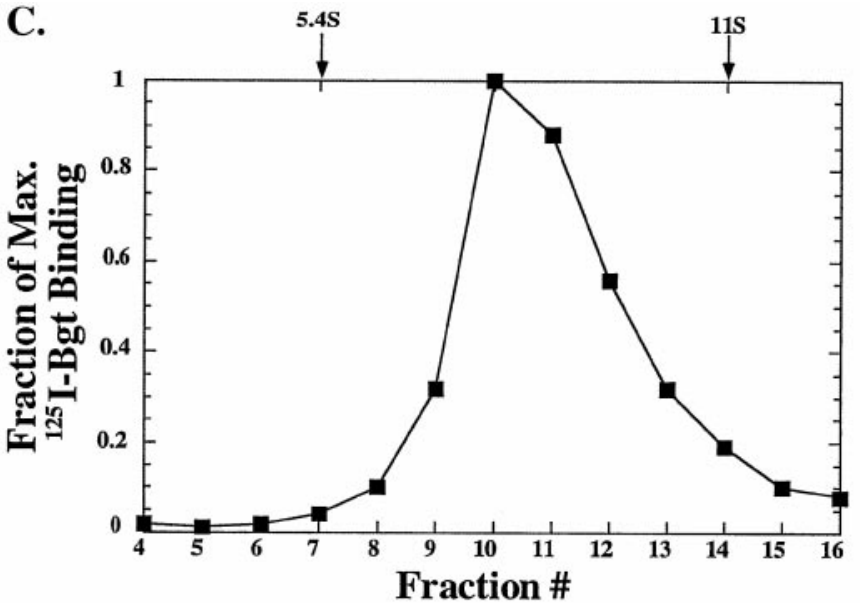
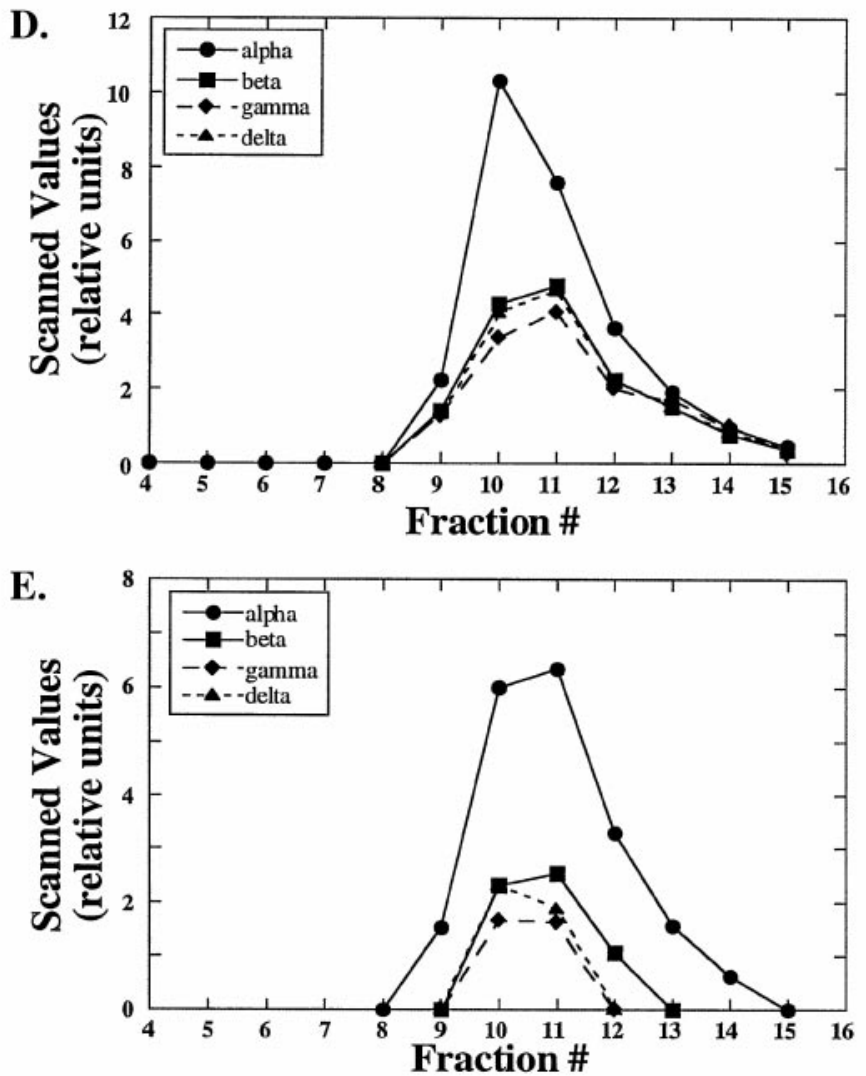
Fraction \#

Figure 4. The second Bgt binding site forms on 9S complexes that are transported to the cell surface. $A$, Metabolically labeled, intracellular AChRs. Cells were pulse-labeled with ${ }^{35} \mathrm{~S}$-methionine-cysteine for 30 min at $37^{\circ} \mathrm{C}$ and cultured for $48 \mathrm{hr}$ at $20^{\circ} \mathrm{C}$. During the chase, the cells were surface-bound with ${ }^{125} \mathrm{I}$-Bgt and solubilized. The number of cell surface ${ }^{125} \mathrm{I}$-Bgt sites was then determined, after which the surface AChRs were immunoprecipitated with anti-Bgt antibodies to separate surface and intracellular AChR complexes. After the immunoprecipitation, the number of precipitated ${ }^{125} \mathrm{I}$-Bgt sites was again determined as well as the number of sites remaining in the cell lysate after the immunoprecipitation. Both were consistent with precipitation of $94 \%$ of the surface counts. Note also that any Bgt-bound AChRs that escaped precipitation, at most $6 \%$, would not be precipitated by the Bgt-Sepharose used to precipitate the intracellular sites in Figure $4 A$. The intracellular AChR complexes that remained in the supernatant after the immunoprecipitation were fractionated on a 5-20\% linear sucrose gradient and precipitated using Bgt-Sepharose. The arrows mark the peak fractions of each of three standards: alkaline phosphatase (5.4S), cell surface Torpedo AChRs (9S, Fig. 4C), and catalase (11S), which were run on parallel gradients. $B$, Metabolically labeled, surface AChRs. Cells were pulselabeled with ${ }^{35} \mathrm{~S}$-methionine-cysteine for $30 \mathrm{~min}$ at $37^{\circ} \mathrm{C}$, cultured for 48 $\mathrm{hr}$ at $20^{\circ} \mathrm{C}$, and then cultured for $2 \mathrm{hr}$ at $37^{\circ} \mathrm{C}$ to increase the transport of AChRs to the cell surface (Ross et al., 1991). During the chase, the cells were surface-bound with ${ }^{125} \mathrm{I}-\mathrm{Bgt}$ and solubilized. Solubilized subunits were fractionated on a 5-20\% linear sucrose gradient, and the surface, Bgt-bound AChRs were immunoprecipitated with anti-Bgt antibodies. $C$, ${ }^{125} \mathrm{I}$-Bgt-bound, surface AChRs. After $48 \mathrm{hr}$ in culture at $20^{\circ} \mathrm{C}$, the cells were surface-bound with ${ }^{125} \mathrm{I}$-Bgt and solubilized. Solubilized Bgt-bound AChRs were fractionated on a 5-20\% linear sucrose gradient. The ${ }^{125} \mathrm{I}-$ Bgt-bound AChRs sedimented in a single peak, which has previously been shown to be centered at 9S (Claudio et al., 1987). The location of arrow for the 9S standard in Figure $4 A$ is based on the sedimentation of the peak fractions (10 and 11) on this gradient. $D$, Scanning densitometry of intracellular AChR subunit. The scanned values of the ${ }^{35}$ S-labeled AChR subunits in Figure $4 A$ are displayed. The signal in fraction 8 was too weak to quantify. The subunit ratio, $\alpha: \beta: \gamma: \delta$, was $1.6: 1: 0.90: 0.95$ in fraction $9,2.4: 1: 0.80: 0.94$ in fraction 10, 1.8:1:0.85:0.98 in fraction 11, 1.6:1:0.91:1.0 in fraction $12,1.2: 1: 1.1: 1.0$ in fraction 13, 1.2:1:1.2:1.0 in fraction 14, and 1.3:1:0.70:1.1 in fraction 15. The scanned values are in arbitrary units. E, Scanning densitometry of surface AChRs. The scanned values of the ${ }^{35}$ S-labeled AChR subunits in Figure $4 B$ are displayed. The $\alpha$ subunit signal could be quantified in fractions $9-14$, the $\beta$ subunit signal in fractions $10-12$, and the $\gamma$ and $\delta$ subunit signal only in fractions 10 and 11 . The subunit ratio, $\alpha: \beta: \gamma: \delta$, was 2.6:1:0.70:1.0 in fraction 10, 2.6:1:0.70:1.0 in fraction 11, and 3.1:1:0:0 in fraction 12. The scanned values are in arbitrary units but are scaled relative to the values in Figure $4 D$ taking into account the difference in the exposure time of the autoradiograms $(6 \mathrm{~d}$ in Fig. $A$ and $5 \mathrm{~d}$ in Fig. $B)$.

\section{DISCUSSION}

\section{ACh binding sites form on subunit complexes other than $\alpha \gamma$ and $\alpha \delta$ heterodimers}

The data presented in this paper contradict the current model in which it is proposed that the two ACh sites form at the same time early in the process of subunit assembly. According to this model, the binding sites appear after $\alpha$ subunits have assembled with $\gamma$ or $\delta$ subunits into $\alpha \gamma$ and $\alpha \delta$ heterodimers. Differences in the two binding sites occur because one binding site forms in the vicinity of the $\alpha \gamma$ subunit interface on the resulting $\alpha \gamma$ heterodimers, and the other site forms in a homologous location on $\alpha \delta$ heterodimers. After ACh binding site formation, $\alpha \gamma$ and $\alpha \delta$ heterodimers assemble with $\beta$ subunits into $\alpha_{2} \beta \gamma \delta$ pentamers (Blount et al., 1990; Gu et al., 1991; Saedi et al., 1991). The evidence in support of this "heterodimer model" is based on experiments in which less than the full complement of AChR subunits were heterologously expressed. When $\alpha$ subunits are expressed alone (Blount and Merlie, 1988; Paulson et al., 1991) or with $\beta$ subunits (Blount and 


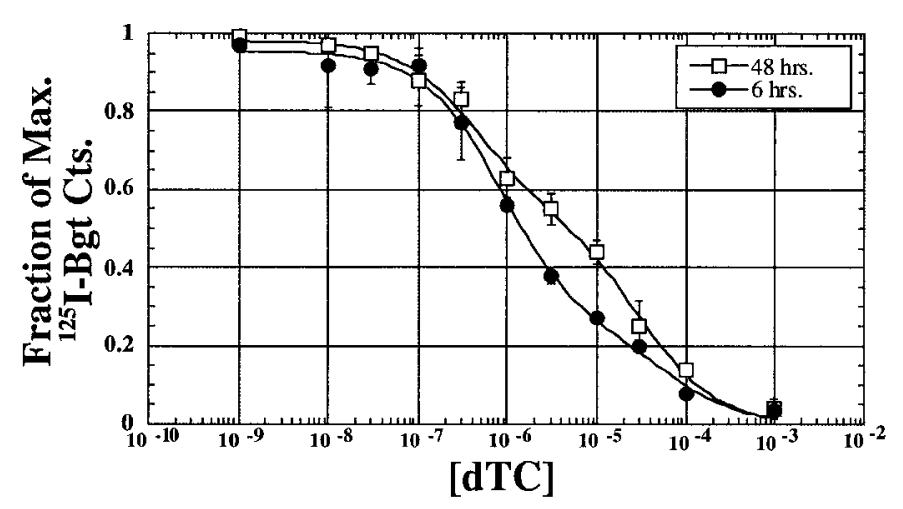

Figure 5. The first ACh site formed has a high affinity for curare. Displayed is the inhibition of the initial rate of ${ }^{125} \mathrm{I}$-Bgt binding to AChR intracellular subunit complexes by dTC. Intracellular subunit complexes were isolated 6 (filled circles) or 48 (open squares) hr after cells were shifted from 37 to $20^{\circ} \mathrm{C}$ by immunoprecipitation with $\mathrm{mAb} 14$. For the AChR subunit complexes isolated $48 \mathrm{hr}$ after the temperature shift, the data were well fit by assuming an equal number of two binding sites with a $\sim 70$-fold difference in the $\mathrm{IC}_{50}, 4.5 \pm 1.4 \times 10^{-7}$ versus $3.0 \pm 0.7 \times$ $10^{-5} \mathrm{M}$, for dTC, as indicated by the line through the data. For the AChR subunit complexes isolated $6 \mathrm{hr}$ after the temperature shift, the data could not be fit by the same model used to fit the $48 \mathrm{hr}$ data. Instead, most of the inhibition by dTC $(\sim 80 \%)$ was well fit assuming a single binding site, as indicated by the line through the data. The $\mathrm{IC}_{50}$ for this site, $8.5 \pm 1.7 \times$ $10^{-7} \mathrm{M}$, was approximately the same as the high-affinity value estimated from the two-site model fit to the $48 \mathrm{hr}$ data. The rest of the sites $(\sim 20 \%)$ were well fit by assuming a single low-affinity binding site for dTC. These data indicate that the first ACh binding site formed, the site found on $\alpha \beta \gamma \delta$ tetramers, is the high-affinity curare site. Each point represents the mean $\pm \mathrm{SD}$ of three determinations, with each determination the mean from ${ }^{125} \mathrm{I}$-Bgt binding to two $6 \mathrm{~cm}$ cultures.

Merlie, 1989), no ACh binding sites form. However, when $\alpha$ subunits are expressed with either $\gamma$ or $\delta$ subunits, ACh binding sites with high affinity for curare form on $\alpha \gamma$ subunit complexes, and ACh binding sites with low affinity for curare form on the $\alpha \delta$ subunit complexes.

It is important to note that there are no disagreements about the data on which the heterodimer model is based. In fact, we obtained similar results when the same combinations of Torpedo AChR subunits were expressed (Green and Claudio, 1993). Contradictions with the heterodimer model only arose when studying the assembly of all four AChR subunits. Two features of our methods allowed us to overcome difficulties previously encountered when isolating AChR assembly intermediates. First, it was critical that $\mathrm{AChR}$ complexes were solubilized in detergent other than Triton X-100, which causes the dissociation of most partially assembled AChR subunit complexes. We used a mixture of Lubrol PX and phosphatidylcholine to solubilize the subunit complexes, and this mixture appears to preserve most of the subunit associations (Green and Claudio, 1993; Green and Wanamaker, 1997). The second feature is the temperature sensitivity of the Torpedo AChR subunit assembly. At $20^{\circ} \mathrm{C}$, the temperature at which experiments were performed in this paper, the rate of assembly is slowed by more than an order of magnitude, greatly aiding the isolation of subunit complexes on which the ACh binding sites form.

Within the error of our measurements, we find no evidence for ACh binding to $\alpha \gamma$ or $\alpha \delta$ heterodimers. All complexes isolated by bromo-ACh affinity purification, Bgt-Sepharose precipitation, or $\mathrm{mAb} 247 \mathrm{~g}$ immunoprecipitation contained $\beta$ subunits. In addition, when size-fractionated on sucrose gradients, the isolated complexes are larger than expected for heterodimer complexes, either $\alpha \beta \gamma$ trimers, $\alpha \beta \gamma \delta$ tetramers, or $\alpha_{2} \beta \gamma \delta$ pentamers (Figs. 3, 4). It is unlikely that that we missed $\alpha \gamma$ and $\alpha \delta$ heterodimers with ACh binding sites using these different techniques. Even though the bromo-ACh affinity purification of the AChR subunits does not quantitatively bind all AChR complexes, the $\alpha \gamma$ and $\alpha \delta$ heterodimers would have to bind $\mathrm{ACh}$ with a lower affinity than the complexes purified for us to have missed these complexes. A number of studies have demonstrated that $\alpha \gamma$ and $\alpha \delta$ complexes bind agonists and antagonists with the same affinity as the fully assembled receptor (Blount and Merlie, 1989), thus eliminating this possibility. Furthermore, although the bromo-ACh affinity purification is not quantitative, precipitations using both BgtSepharose and mAb $247 \mathrm{~g}$ are quantitative; that is, we precipitate just as much of the assembling subunit complexes with the BgtSepharose and mAb $247 \mathrm{~g}$ as with any other subunit-specific antisera. Because Bgt binding sites and the mAb 247g epitope appear to overlap with $\mathrm{ACh}$ binding sites on $\alpha \gamma$ and $\alpha \delta$ dimers, both techniques should have precipitated any $\alpha \gamma$ and $\alpha \delta$ dimers. Finally, previous studies from our laboratory using different subunit-specific and conformation-dependent antibodies never found evidence for the assembly of $\alpha \gamma$ and $\alpha \delta$ heterodimers in cells expressing the all four AChR subunits, neither Torpedo $\mathrm{AChR}$ subunits at $20^{\circ} \mathrm{C}$ nor mouse $\mathrm{AChR}$ subunits at $37^{\circ} \mathrm{C}$ (Green and Claudio, 1993; Green and Wanamaker, 1997). Thus, the results do not appear to be an artifact attributed to the temperature or species specificity.

\section{Subunit association sites for the $\delta$ and second $\alpha$ subunits}

Contrary to the heterodimer model, we found that the two distinguishable ACh binding sites form at different times. While the AChR is still assembling, the first ACh binding site forms on $\alpha_{\gamma}$ subunits apparently in $\alpha \beta \gamma \delta$ tetramers. After completion of subunit assembly, the second site forms on $\alpha_{\delta}$ subunits in $\alpha_{2} \beta \gamma \delta$ pentamers. These findings, together with our data that the first Bgt binding site and the mAb 14 epitope appear on $\alpha \beta \gamma$ trimers (Fig. $3 A, C$ ), are in agreement with "the sequential model" of AChR assembly that we previously proposed (Green and Claudio, 1993). As shown in Figure $7 A$, the sequential model is consistent with two different pathways, which can be distinguished based on the order in which the two ACh binding sites form. Evidence was presented that the first ACh binding site formed is the $\alpha_{\gamma}$ site and the second ACh binding site is the $\alpha_{\delta}$ site. AChRs assemble, therefore, along path 2, with the $\delta$ subunit assembling at the interface between the $\gamma$ and $\beta$ subunits and the second $\alpha$ subunit assembling at the interface between the $\gamma$ and $\delta$ subunits.

Although the two unassembled subunits associate between different pairs of subunits, both associate with the same $\gamma$ subunit interface, the interface in where the $\beta$ subunit also associates during the assembly of the $\alpha \beta \gamma$ trimer (Fig. $7 B$ ). This region of the $\gamma$ subunit appears to play a critical role in the oligomerization process, because it is the only subunit interface that accepts different unassembled subunits during assembly. Our data suggest that this region changes subunit specificity to accommodate the three different subunits at different times during assembly.

The formation of the two ACh binding sites at different times and on different AChR subunit complexes may be a factor contributing to the differences between the two ACh binding sites. It is thought that the two AChR agonist binding sites are distinguishable because the two $\alpha$ subunits interface with different 

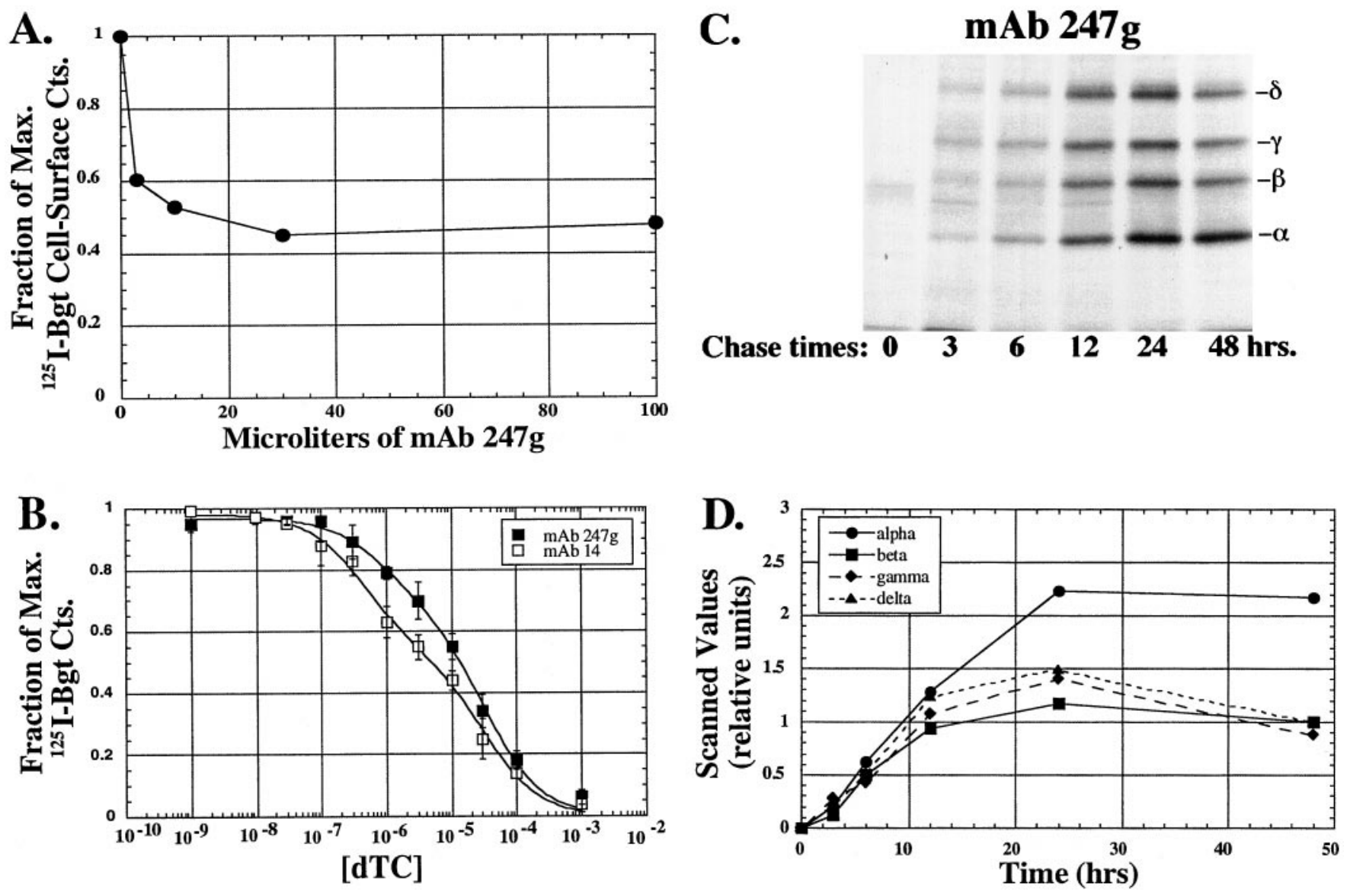

Figure 6. The first ACh binding site forms on the $\alpha_{\gamma}$ subunit. A, mAb $247 \mathrm{~g}$ binds to and blocks one of the two Bgt binding sites. The indicated amounts of $\mathrm{mAb} 247 \mathrm{~g}$ were added to the medium of intact cells for $2 \mathrm{hr}$ at $20^{\circ} \mathrm{C}$, after which intact cells were bound by ${ }^{125} \mathrm{I}$-Bgt to determine the number of cell surface Bgt sites. At saturating concentrations, $\mathrm{mAb} 247 \mathrm{~g}$ blocked $\sim 50 \%$ of the cell surface Bgt sites. Each point represents the mean from ${ }^{125} \mathrm{I}$-Bgt binding to two $6 \mathrm{~cm}$ cultures. $B$, mAb $247 \mathrm{~g}$ binds to the high-affinity curare binding site. Displayed is the inhibition of the initial rate of ${ }^{125} \mathrm{I}$-Bgt binding to AChR intracellular subunit complexes by dTC. Forty-eight hours after cells were shifted from 37 to $20^{\circ} \mathrm{C}$, intracellular subunit complexes were isolated by immunoprecipitation with mAb $247 \mathrm{~g}$ ( filled squares). Also displayed for comparison are the data from Figure 5 in which intracellular subunit complexes isolated by immunoprecipitation with $\mathrm{mAb} 14,48 \mathrm{hr}$ after cells were shifted from 37 to $20^{\circ} \mathrm{C}$ (open squares). The inhibition by dTC obtained for the complexes precipitated by mAb $247 \mathrm{~g}$ was fit by the two-site model used to fit the mAb 14 data. Most of the inhibition by dTC ( $\sim 75 \%)$ was well fit, assuming a single binding site as indicated by the line through the data. The $\mathrm{IC}_{50}$ for this site, $3.2 \pm 0.6 \times 10^{-5} \mathrm{M}$, was approximately the same as the low-affinity value estimated from the two-site model fit to the $48 \mathrm{hr}$ data. The rest of the sites ( $\sim 25 \%)$ were well fit by assuming a single high-affinity binding site for dTC. Each point represents the mean \pm SD of three determinations, with each determination the mean from ${ }^{125}$ I-Bgt binding to two 6 $\mathrm{cm}$ cultures. $C, \mathrm{mAb} 247 \mathrm{~g}$ precipitation of labeled subunits. Cells were treated as in Figures $1 A$ and $2 A$, except that AChR subunits were immunoprecipitated with mAb $247 \mathrm{~g}$. $D$, Scanning densitometry of AChR subunits precipitated by mAb $247 \mathrm{~g}$. The time course of the formation of the $\mathrm{mAb} 247 \mathrm{~g}$ epitope is shown quantitatively from the scanned values of the experiment shown in $C$.

subunits, either $\gamma$ or $\delta$ subunits (Blount and Merlie, 1989; Pedersen and Cohen, 1990; Sine and Claudio, 1991). Recently, however, we have found differences in the agonist binding sites of another AChR subtype consisting of subunits derived from the same gene product (Rangwala et al., 1997). The distinctions between the agonist binding sites of this homomeric AChR cannot arise because of different "structural" subunits interfacing with the ligand-binding subunits. An alternative mechanism must be causing the differences in the ligand binding sites. Our results suggest that differences in the homomeric AChR ligand binding sites could be caused by asymmetries in the process by which the ligand binding sites form. Asymmetries, such as the ligand binding sites forming at different times in the assembly process or on AChR subunit complexes of different sizes, could give rise to differences in ligand binding sites even though all the subunits derive from the same gene product. It is further possible that the same asymmetries are contributing to the distinctions between the two ACh binding sites on the muscle-type AChR.

\section{Subunit folding during and after AChR assembly}

This study provides additional evidence that the AChR subunits continue to fold during subunit assembly and new evidence that subunit folding continues after all of the subunits have assembled together into pentamers. Previously, we have shown that subunit folding events, such as the formation of the first Bgt binding site and the mAb 14 epitope, appear to be the rate-limiting steps in the assembly of the AChR pentamer (Green and Claudio, 1993), and that prevention of these or other folding events can block subunit assembly at different stages (Green and Wanamaker, 1997). As diagrammed in Figure $7 B$, we envision that these folding events underlie the subunit rearrangements on the $\alpha \beta \gamma$ trimers and $\alpha \beta \gamma \delta$ tetramers that change the subunit specificity at the sites in which the unassembled $\delta$ and second $\alpha$ subunits associate. The formation of the ACh binding sites appears to be different from other folding events in that they immediately follow the assembly of the $\delta$ and second $\alpha$ subunits and do not 
A.

Path 1
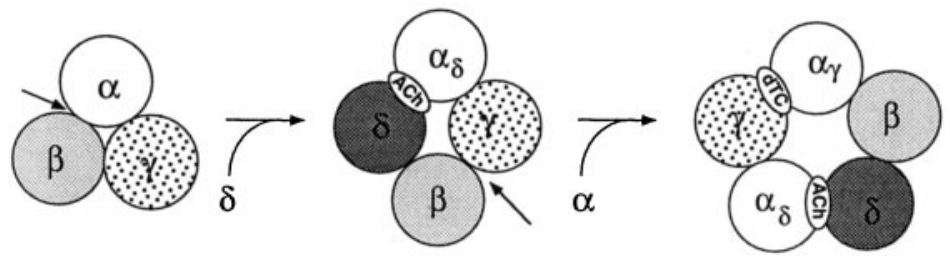

Path 2
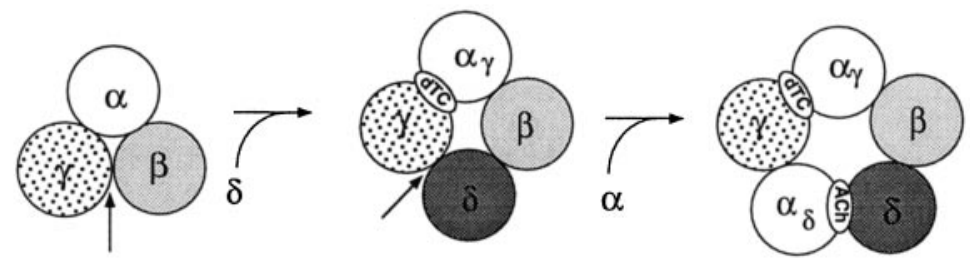

B.

\title{
Subunit Folding and Oligomerization Events (1st Set)
}

\author{
The 1st ACh \\ Binding Site Forms
}

\section{Subunit Folding and Oligomerization Events (2nd Set)}
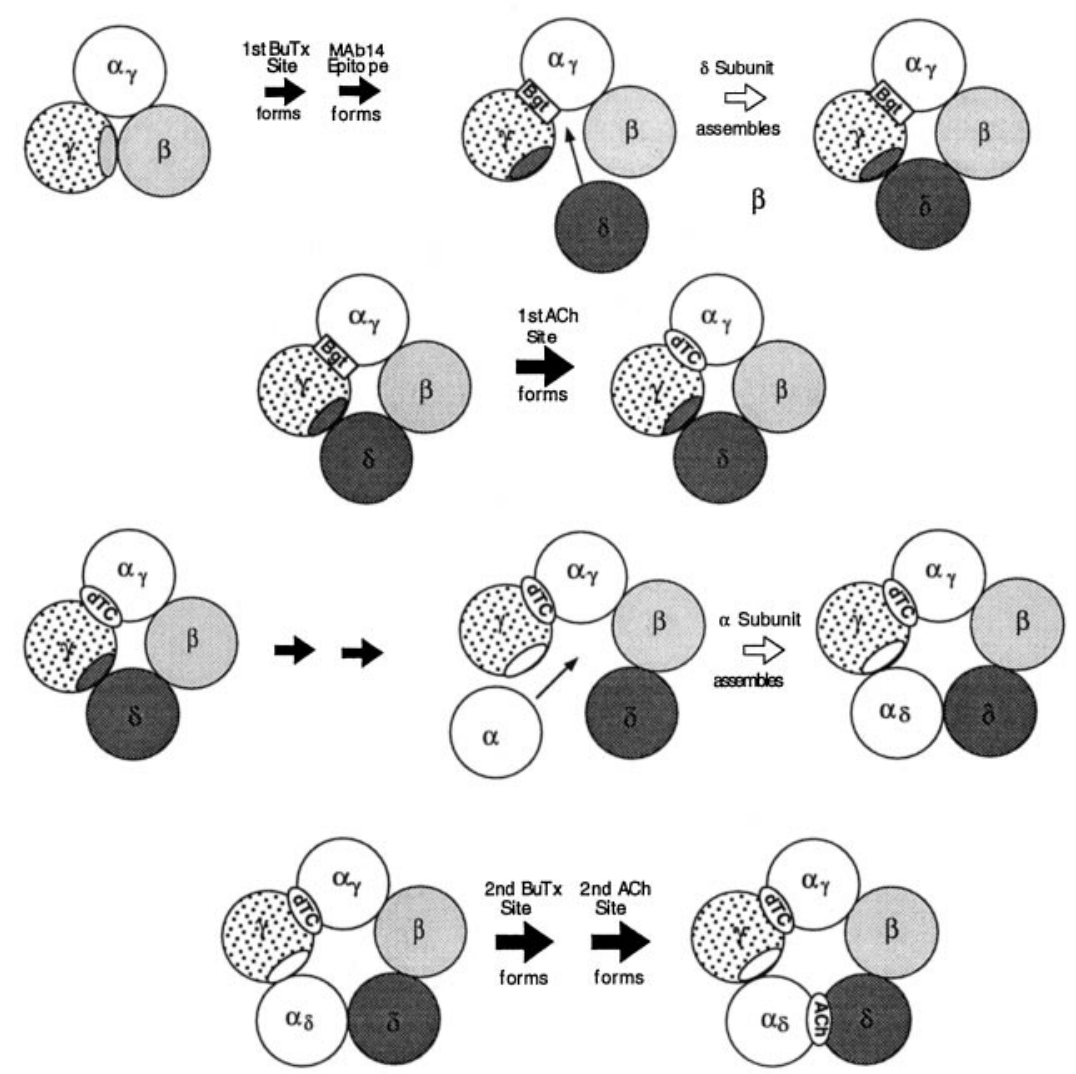

Figure 7. A, The order of ACh binding site formation distinguishes between two different paths of AChR subunit assembly. Displayed are two different paths of AChR subunit assembly consistent with our previous studies (Green and Claudio, 1993; Green and Wanamaker, 1997). The two paths differ where the $\delta$ and second $\alpha$ subunits associate (marked by arrows) and where the two different ACh binding sites, the high-affinity dTC site (marked as $d T C$ ) or the low-affinity dTC site (marked as $A C h$ ), form first. AChR subunit assembly along path 2 is demonstrated by our finding that the $\alpha_{\gamma}$ ACh binding site forms first. The subunit arrangement of the $\alpha_{2} \beta \gamma \delta$ pentamer shown in both paths is the favored arrangement (Karlin et al., 1983; Blount and Merlie, 1989; Pedersen and Cohen, 1990; Sine and Claudio, 1991; Machold et al., 1995). Another subunit arrangement of the $\alpha_{2} \beta \gamma \delta$ pentamer has been proposed (Kubalek et al., 1987), in which the positions of the $\beta$ and $\gamma$ subunits in the pentamer are switched. However, the positions of the arrows do not change with this arrangement, even though the positions of the $\beta$ and $\gamma$ subunits change. $B$, The subunit folding and oligomerization events that precede ACh binding site formation. Diagrammed are the subunit folding and oligomerization events that precede formation of both ACh binding sites. Subunit folding events are denoted by the filled arrows, and oligomerization events are denoted by the open arrows. Formation of the first Bgt binding site and the mAb 14 epitope precede the addition of the $\delta$ subunit and the formation of the first ACh binding site, as described in the Discussion. Other folding events, not described in this paper, appear to precede the addition of the second $\alpha$ subunit and the formation of the second ACh binding site (Green and Wanamaker, 1997). Our results indicate that the folding events lead to a change in the subunit specificity at the sites in which the unassembled $\delta$ and second $\alpha$ subunits associate. This change in the subunit specificity is shown schematically by the change in the shading of the $\gamma$ subunit region that contributes to the interfaces in which the different unassembled subunits associate.

contribute to any change in the sites in which unassembled subunits assemble. Previously it was shown that AChR complexes continue to reside in the endoplasmic reticulum (ER) after pentamers have assembled (Ross et al., 1991). The formation of the second $\mathrm{ACh}$ and Bgt binding sites after assembly of the AChR pentamer suggests that the ER "quality control" mechanisms (Hurtley and Helenius, 1989) recognize and selectively retain pentamers lacking the second $\mathrm{ACh}$ and Bgt binding sites. Because 
activation of the AChR requires formation of both ACh binding sites (Cash and Hess, 1980; Sine and Taylor, 1980), the AChR is not functional until after assembly of the $\alpha_{2} \beta \gamma \delta$ pentamer, and it is an intriguing possibility that $\mathrm{AChR}$ activation serves as the signal for receptor release from the ER.

\section{REFERENCES}

Blount P, Merlie JP (1988) Native folding of an acetylcholine receptor $\alpha$ subunit expressed in the absence of other receptor subunits. J Biol Chem 263:1072-1080.

Blount P, Merlie JP (1989) Molecular basis of the two nonequivalent ligand binding sites of the muscle nicotinic acetylcholine receptor. Neuron 3:349-357.

Blount P, Smith MM, Merlie JP (1990) Assembly intermediates of the mouse muscle nicotinic acetylcholine receptor in stably transfected fibroblasts. J Cell Biol 111:2601-2611.

Cash DJ, Hess GP (1980) Molecular mechanisms of acetylcholine receptor-controlled ion translocation across cell membranes. Proc Natl Acad Sci USA 77:842-846.

Chak A, Karlin A (1992) Purification and reconstitution of nicotinic acetylcholine receptor. Methods Enzymol 207:546-555.

Changeux JP (1995) Thudichum medal lecture. The acetylcholine receptor: a model for allosteric membrane proteins. Biochem Soc Trans 23:195-205.

Claudio T, Green WN, Hartman DS, Hayden D, Paulson HL, Sigworth FJ, Sine SM, Swedlund A (1987) Genetic reconstitution of functional acetylcholine receptor channels in mouse fibroblasts. Science 238:1688-1694.

Galzi JL, Changeux J-P (1994) Neurotransmitter-gated ion channels as unconventional allosteric proteins. Curr Opin Struct Biol 4:554-565.

Green WN, Claudio T (1993) Acetylcholine receptor assembly: subunit folding and oligomerization occur sequentially. Cell 74:57-69.

Green WN, Wanamaker CP (1997) The role of the cysteine loop in acetylcholine receptor assembly. J Biol Chem 272:20945-20953.

Gu Y, Forsayeth JR, Verrall S, Yu XM, Hall ZW (1991) Assembly of mammalian muscle acetylcholine receptors in transfected COS cells. J Cell Biol 114:799-807.

Hartman DS, Poo M-M, Green WN, Ross AF, Claudio T (1990) Synaptic contact between embryonic neurons and acetylcholine receptorfibroblasts. J Physiol (Lond) 84:42-49.

Hurtley SM, Helenius A (1989) Protein oligomerization in the endoplasmic reticulum. Annu Rev Cell Biol 5:277-307.

Karlin A, Akabas MH (1995) Toward a structural basis for the function of nicotinic acetylcholine receptors and their cousins. Neuron 15:1231-1244.

Karlin A, Holtzman E, Yodh N, Lobel P, Wall J, Hainfeld J (1983) The arrangement of the subunits of the acetylcholine receptor of Torpedo californica. J Biol Chem 258:6678-6681.

Kreienkamp HJ, Maeda RK, Sine SM, Taylor P (1995) Intersubunit contacts governing assembly of the mammalian nicotinic acetylcholine receptor. Neuron 14:635-644.

Kubalek E, Ralston S, Lindstrom J, Unwin N (1987) Location of subunits within the acetylcholine receptor by electron image analysis of tubular crystals from Torpedo marmorata. J Cell Biol 105:9-18.
Lindstrom JM (1995) Nicotinic acetylcholine receptors. In: Handbook of receptors and channels. Ligand- and voltage-gated ion channels. (North RA, ed), pp 153-175. Boca Raton, FL: CRC.

Machold J, Weise C, Utkin Y, Tsetlin V, Hucho F (1995) The handedness of the subunit arrangement of the nicotinic acetylcholine receptor from Torpedo californica. Eur J Biochem 234:427-430.

Merlie JP, Lindstrom J (1983) Assembly in vivo of mouse muscle acetylcholine receptor: identification of an $\alpha$ subunit species that may be an assembly intermediate. Cell 34:747-757.

Mihovilovic M, Richman DP (1987) Monoclonal antibodies as probes of the $\alpha$-Bungarotoxin and cholinergic binding regions of the acetylcholine receptor. J Biol Chem 262:4978-4986.

Mishina M, Tobimatsu T, Tanaka K, Fujita Y, Fukuda K, Kurasake M, Takahashi H, Morimoto Y, Hirose T, Inayama S, Takahasi T, Kuno M, Numa S (1985) Location of functional regions of acetylcholine receptor a-subunit by site-directed mutagenesis. Nature 313:364-369.

Neubig RR, Cohen JB (1979) Equilibrium binding of $\left[{ }^{3} \mathrm{H}\right]$ tubocurarine and $\left[{ }^{3} \mathrm{H}\right]$ acetylcholine by Torpedo postsynaptic membranes: stoichiometry and ligand interactions. Biochemistry 18:5464-5475.

Paulson HL, Ross AF, Green WN, Claudio T (1991) Analysis of early events in acetylcholine receptor assembly. J Cell Biol 113:1371-1384.

Pedersen SE, Cohen JB (1990) $d$-Tubocurarine binding sites are located at $\alpha \gamma$ and $\alpha \delta$ subunit interfaces of the nicotinic acetylcholine receptor. Proc Natl Acad Sci USA 87:2785-2789.

Rangwala F, Drisdel RC, Rakhilin S, Ko E, Atluri P, Harkins AB, Fox AP, Salman SB, Green WN (1997) Neuronal $\alpha$-Bungarotoxin receptors differ structurally from other nicotinic acetylcholine receptors. J Neurosci 17:8201-8212.

Ross AF, Green WN, Hartman DS, Claudio T (1991) Efficiency of acetylcholine receptor subunit assembly and its regulation by cAMP. J Cell Biol 113:623-636.

Saedi MS, Conroy WG, Lindstrom J (1991) Assembly of Torpedo acetylcholine receptors in Xenopus oocytes. J Cell Biol 112:1007-1015.

Sine S, Claudio T (1991) $\gamma$ - and $\delta$-subunits regulate the affinity and the cooperativity of ligand binding to the acetylcholine receptor. J Biol Chem 266:19369-19377.

Sine SM, Taylor P (1980) The relationship between agonist occupation and the permeability response of the cholinergic receptor revealed by bound cobra $\alpha$-toxin. J Biol Chem 255:10144-10156.

Sine SM, Taylor P (1981) Relationship between reversible antagonist occupancy and the functional capacity of the acetylcholine receptor J Biol Chem 256:6692-6699.

Tsigelny I, Sugiyama N, Sine SM, Taylor P (1997) A model of the nicotinic receptor extracellular domain based on sequence identity and residue location. Biophys J 73:52-66.

Tzartos SJ, Lindstrom JM (1980) Monoclonal antibodies used to probe acetylcholine receptor structure: localization of the main immunogenic region and detection of similarities between subunits. Proc Natl Acad Sci USA 77:755-759.

Unwin N (1993) Neurotransmitter action: opening of ligand-gated ion channels. Cell 72:31-41.

Wang YD, Claudio T (1993) Xenopus muscle acetylcholine receptor $\alpha$ subunits bind ligands with different affinities. J Biol Chem 268:1878218793. 